\title{
Deep optical observations of G $65.3+5.7$
}

\author{
F. Mavromatakis ${ }^{1}$, P. Boumis ${ }^{1}$, J. Papamastorakis ${ }^{1,2}$, and J. Ventura ${ }^{1}$ \\ 1 University of Crete, Physics Department, PO Box 2208, 71003 Heraklion, Crete, Greece \\ 2 Foundation for Research and Technology-Hellas, PO Box 1527, 71110 Heraklion, Crete, Greece
}

Received 12 February 2002 / Accepted 3 April 2002

\begin{abstract}
We present the first CCD mosaic of the supernova remnant G $65.3+5.7$ in the optical emission lines of [O II] and [O III]. The new images reveal several diffuse and filamentary structures both inside and outside the extent of the remnant as defined by its $\mathrm{X}$-ray and radio emission. The medium ionization line of [O III] $5007 \AA$ provides the sharpest view to the system, while the remnant appears less filamentary in the emission line of [O II]. There are significant morphological differences between the two images strongly suggesting the presence of incomplete shock structures. Deep long-slit spectra were taken at several different positions of G 65.3+5.7. All spectra originate from shock heated gas, while the majority of them is characterized by large $[\mathrm{O} \mathrm{III}] / \mathrm{H} \beta \mathrm{ratios}$. The sulfur line ratios indicate electron densities below $\sim 200 \mathrm{~cm}^{-3}$, while estimates of the shock velocities lie in the range of $90-140 \mathrm{~km} \mathrm{~s}^{-1}$. Finally, the observed variations of the $\mathrm{H} \alpha / \mathrm{H} \beta$ ratios may reflect the presence of intrinsic absorption affecting the optical spectra.
\end{abstract}

Key words. ISM: general - ISM: supernova remnants - ISM: individual objects: G 65.3+5.7

\section{Introduction}

Gull et al. (1977) reported the detection of a new supernova remnant in Cygnus during an optical survey with narrow band filters. In particular, they obtained an [O III] photograph showing the existence of a $\sim 3.3 \times 4.0$ filamentary shell. Due to its large extent, high resolution imagery has been performed only in selected regions of the remnant (Fesen et al. 1983 and references therein). Subsequent, optical spectra obtained by Sabbadin \& D'Odorico (1976) and Fesen et al. (1985) suggested emission from shock heated gas. A stratification among lower and medium ionization lines has been reported by Sitnik et al. (1983) for another area of G 65.3+5.7. Fesen et al. (1985) obtained long-slit spectra at two different positions of G 65.3+5.7 showing strong sulfur line emission relative to $\mathrm{H} \alpha$ and especially one of the spectra was characterized by very strong $[\mathrm{O} \mathrm{III}]$ emission compared to $\mathrm{H} \beta(\sim 40)$. High $[\mathrm{O} \mathrm{III}] / \mathrm{H} \beta$ ratios have been observed in other remnants as well and imply the presence of incomplete recombination zones (e.g. Raymond et al. 1988). Radio observations at $1420 \mathrm{MHz}$ (Reich et al. 1979) confirmed the non-thermal nature of the radio emission from this shell and found a spectral index of $\sim 0.61$. Current estimates of the linear diameter and the distance to the remnant are $\sim 70 \mathrm{pc}$

Send offprint requests to: F. Mavromatakis,

e-mail: fotis@physics.uoc.gr and 0.9-1.0 kpc, respectively (Gull et al. 1977; Reich et al. 1979; Rosado 1981; Sitnik et al. 1983). Soft X-ray observations show that G $65.3+5.7$ is a weak source of $\mathrm{X}$-ray emission and imply a shock velocity in the range of a few hundred $\mathrm{km} \mathrm{s}^{-1}$ and an age of $\sim 20000$ yrs (Snyder et al. 1978; Mason et al. 1979; Seward 1990). G 65.3+5.7 has also been observed by ROSAT both during the AllSky survey (Aschenbach 1994) and in pointed mode (Lu \& Aschenbach 2002). The analysis of these data showed that the $\mathrm{X}$-ray emission is clumpy and that the forward shock is traveling with a velocity of $\sim 400 \mathrm{~km} \mathrm{~s}^{-1}$ about $26000 \mathrm{yrs}$ after the supernova explosion, while the explosion energy and the interstellar medium density are $\sim 2 \times 10^{50} \mathrm{erg}$ and $\sim 0.02 \mathrm{~cm}^{-3}$, respectively. These parameters were derived assuming a distance to the remnant of $1 \mathrm{kpc}$. Gorham et al. (1996) have performed a pulsar search of several known SNRs at 430 and $1420 \mathrm{MHz}$. Their observations were sensitive to pulsars with periods greater than $1 \mathrm{~ms}$, and flux densities as low as $0.2 \mathrm{mJy}$ but no pulsar was found to be associated with $\mathrm{G} 65.3+5.7$ down to these limits.

In order to study in detail this extended and nearby supernova remnant we performed deep optical imaging observations in the low and medium ionization lines of $[\mathrm{O}$ II $] 3727 \AA$ and $[\mathrm{O}$ III] $5007 \AA$. We have also obtained flux calibrated images of a specific field of $\mathrm{G} 65.3+5.7$ in $\mathrm{H} \alpha+[\mathrm{NII}],[\mathrm{SII}],[\mathrm{OII}]$ and $[\mathrm{O} \mathrm{III}]$. The imaging 
observations are supplemented by deep optical long-slit spectra at 8 different locations of $\mathrm{G} 65.3+5.7$. Information about the observations and the data reduction is given in Sect. 2. In Sects. 3-5 we present the results of our imaging observations and the long-slit spectra. Finally, in Sect. 6 we discuss the physical properties of the remnant.

\section{Observations}

\subsection{Optical images}

The observations presented in this paper were performed with the $0.3 \mathrm{~m}$ Schmidt Cassegrain telescope at Skinakas Observatory, Crete, Greece. The supernova remnant G 65.3+5.7 was observed on July 22, 23 and 24, 2001. A $1024 \times 1024$ SITe CCD was used which had a pixel size of $24 \mu \mathrm{m}$ resulting in a $89^{\prime} \times 89^{\prime}$ field of view and an image scale of $5^{\prime \prime}$ per pixel. Several pointings were performed in order to cover the extended area of G 65.3+5.7. Each of the nine (9) different fields was observed for $2400 \mathrm{~s}$. All fields were projected to a common origin on the sky and were subsequently combined to create the final mosaics in [O II] and [O III]. The astrometric calculations utilized the HST Guide star catalog (Lasker et al. 1999). In addition, we obtained flux calibrated images of a specific field of $\mathrm{G} 65.3+5.7$ in $\mathrm{H} \alpha+[\mathrm{N} \mathrm{II}]$, [S II], [O II] and [O III]. The exposure time of these images is $1800 \mathrm{~s}$ with the exception of the $[\mathrm{O} \mathrm{II}]$ image where we obtained two images, $1800 \mathrm{~s}$ each. This observation was performed in July 13, 1999 with the same telescope and hardware setup as the latest observations. The filter characteristics can be found in Mavromatakis et al. (2000). All coordinates quoted in this work refer to epoch 2000.

We employed standard IRAF and MIDAS routines for the reduction of the data. Individual frames were bias subtracted and flat-field corrected using well exposed twilight flat-fields. The spectrophotometric standard stars HR 5501, HR 8634 and HR 7950 were used for absolute flux calibration in the 1999 observation of G 65.3+5.7.

\subsection{Optical spectra}

Long-slit spectra were obtained on July 15, 1999, and July 24-28, 2001 using the $1.3 \mathrm{~m}$ Ritchey-Cretien telescope at Skinakas Observatory. The data were taken with a 1300 line $\mathrm{mm}^{-1}$ grating and a $800 \times 2000$ Site CCD covering the range of $4750 \AA-6815 \AA$. The slit had a width of $7 ! .7$ and a length of $7 . .9$ and, in all cases, was oriented in the south-north direction. The coordinates of the slit centers along with the number of spectra and their individual exposure times are given in Table 1 . The spectrophotometric standard stars HR 5501, HR 7596, HR 7950, HR 9087, and HR 718 were observed in order to calibrate the spectra.
Table 1. Log of the spectroscopic observations.

\begin{tabular}{lll}
\hline \hline Slit centers & & No of spectra \\
\hline$\alpha($ epoch 2000) & $\delta$ (epoch 2000) & $($ Exp. time) \\
$19^{\mathrm{h}} 42^{\mathrm{m}} 55.3^{\mathrm{s}}$ & $31^{\circ} 43^{\prime} 39^{\prime \prime}$ (Area 1) & $2^{a}(3900)^{b}$ \\
$19^{\mathrm{h}} 40^{\mathrm{m}} 00.1^{\mathrm{s}}$ & $29^{\circ} 59^{\prime} 59^{\prime \prime}$ (Area 2) & $2^{a}(3900)^{b}$ \\
$19^{\mathrm{h}} 31^{\mathrm{m}} 45.0^{\mathrm{s}}$ & $29^{\circ} 34^{\prime} 55^{\prime \prime}$ (Area 3) & $2^{a}(3900)^{b}$ \\
$19^{\mathrm{h}} 27^{\mathrm{m}} 45.7^{\mathrm{s}}$ & $31^{\circ} 30^{\prime} 10^{\prime \prime}$ (Area 4) & $1^{a}(3600)^{b}$ \\
$19^{\mathrm{h}} 28^{\mathrm{m}} 00.9^{\mathrm{s}}$ & $32^{\circ} 16^{\prime} 59^{\prime \prime}$ (Area 5) & $2^{a}(3900)^{b}$ \\
$19^{\mathrm{h}} 35^{\mathrm{m}} 34.0^{\mathrm{s}}$ & $32^{\circ} 54^{\prime} 32^{\prime \prime}$ (Area 6) & $2^{a}(3900)^{b}$ \\
$19^{\mathrm{h}} 38^{\mathrm{m}} 43.0^{\mathrm{s}}$ & $32^{\circ} 28^{\prime} 48^{\prime \prime}$ (Area 7) & $2^{a}(3900)^{b}$ \\
$19^{\mathrm{h}} 35^{\mathrm{m}} 59.2^{\mathrm{s}}$ & $30^{\circ} 12^{\prime} 35^{\prime \prime}$ (Area 8) & $3^{a}(2000)^{b}$ \\
\hline
\end{tabular}

a Number of spectra obtained.

b Exposure time of individual spectra in $\mathrm{s}$.

\section{Imaging of G $65.3+5.7$}

\subsection{The $[\mathrm{O} I 1]$ and $[\mathrm{O} I I]$ line mosaics}

The wide field covered by G $65.3+5.7$ contains a number of extended sources like bright nebulae (e.g. LBN 064.90+06.74; Lynds 1965), dark nebulae (e.g. LDN 817, LDN 816; Lynds 1965) as well as a few planetary nebulae (e.g. PN G064.7+05.0, PN G065.4+03.1). In Fig. 1 we show the mosaic in the low ionization line of [O II] along with letters designating the areas which are described in the text in detail. The [O II] image appears generally less filamentary than the [O III] image (Fig. 2). In addition, the former image contains more diffuse emission than the latter. We start the detailed morphological study of G 65.3+5.7 in these filters from area A (Fig. 1). In this area diffuse emission is seen in the [O II] image, while strong filamentary emission is detected in the medium ionization line image of [O III]. The filament is $\sim 16^{\prime}$ long and its typical projected width is $\sim 1^{\prime}$. Immediately to the south-west of area A we find area B which contains the bright binary $\phi$ Cyg and has been studied by Sitnik et al. (1983). They observed a narrow field around $\phi$ Cyg $(\sim 6.5 \times 6.5)$ and found a stratification in the emission from the $[\mathrm{O} \mathrm{III}],[\mathrm{N} \mathrm{II}]$ and $[\mathrm{S} \mathrm{II}]$ lines with the [O III] emission lying closer to the outer edge of the remnant. Here, the [O II] emission appears more filamentary than the [O III] emission. The $\sim 37^{\prime}$ long filament seen in $[\mathrm{O} \mathrm{II}]$ is broader in the south and becomes narrower as it extends further to the north, while the [O III] image shows roughly a constant projected thickness. The projected thickness close to the northern tip of the [O II] filament is $\sim 32^{\prime \prime}$, while at the same location the thickness of the [O III] filament is $\sim 65^{\prime \prime}$. We also observe here an increasing offset in the outer boundaries of the $[\mathrm{O} \mathrm{II}]$ and [O III] images with the [O III] emission leading and the [O II] emission following. Further to the west we come across area $\mathrm{C}$ for which we have performed flux calibrated imaging observations. This area will be discussed in the next section separately. Significant differences between the low and medium ionization images are found in area D. There are two locations where the [O II] emission is quite intense and 


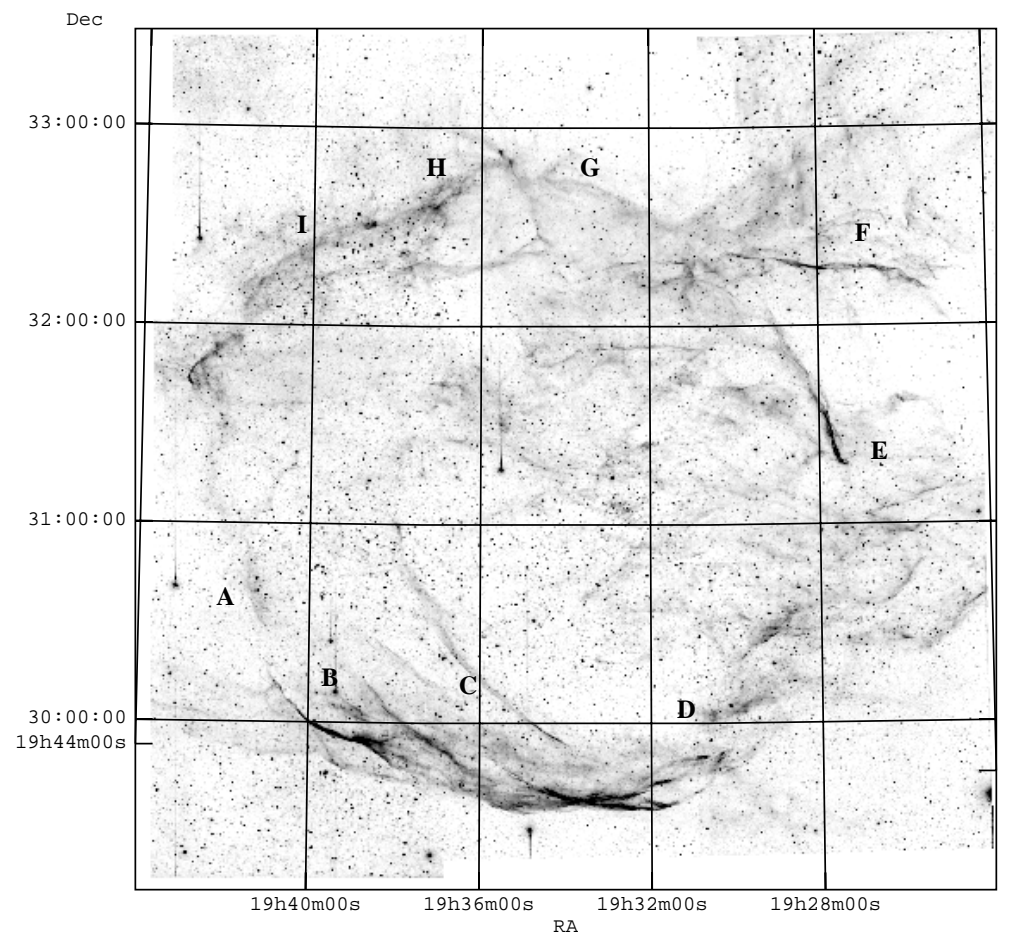

Fig. 1. The 4.3 square field covering the supernova remnant G 65.3+5.7 imaged in the low ionization line of [O II] $3727 \AA$. The letters define the areas which are discussed in the text in more detail.

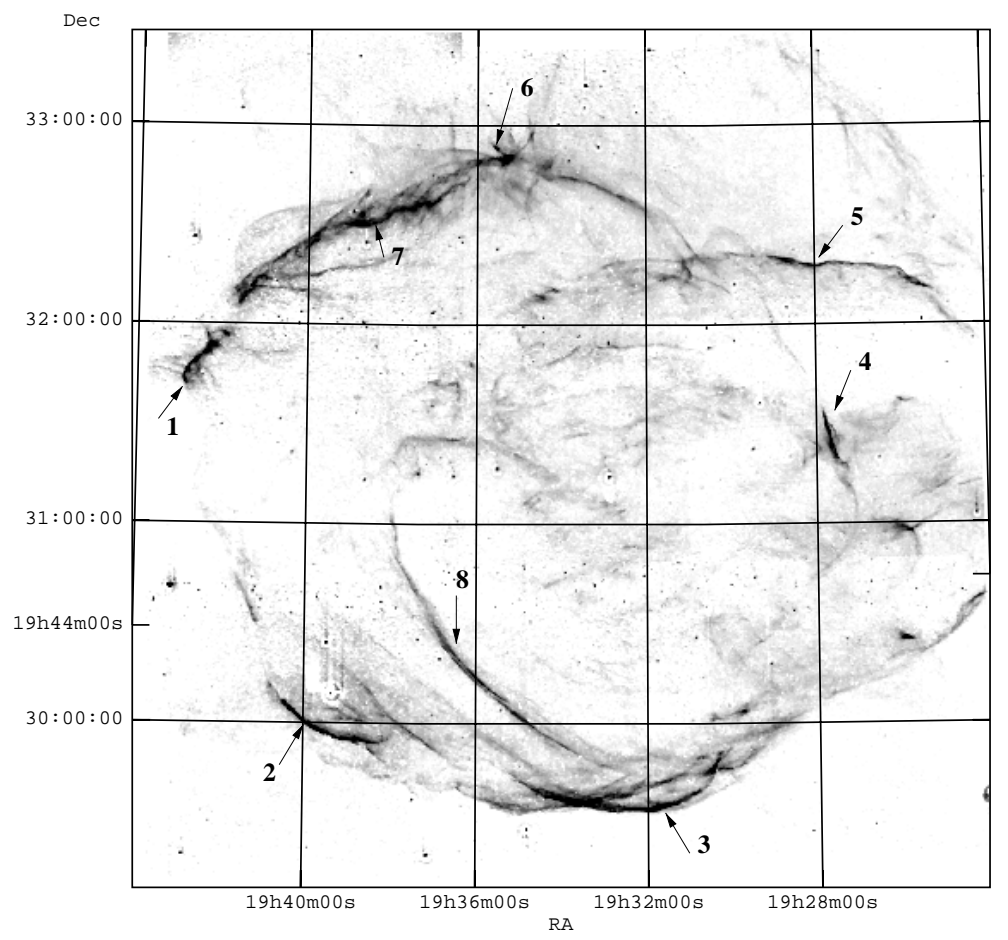

Fig. 2. The remnant G $65.3+5.7$ as it appears in the medium ionization line of [O III] $5007 \AA$. The filamentary nature is clearly seen in this image with respect to the lower ionization image of [O II] (Fig. 1). The arrows roughly point to the projected center on the sky of the slit in each location given in Table 1, while the numbers designate the individual locations.

filamentary, while at the same location the [O III] emission is diffuse and weak. In this area there is also a location where the inverse is observed, i.e. filamentary emission in $[\mathrm{O}$ III $]$ and diffuse, very weak emission in $[\mathrm{O}$ II]. Area E contains a complex network of filaments and diffuse emission in $[\mathrm{OII}]$, while the $[\mathrm{O} \mathrm{III}]$ image provides a sharper view of the area. LBN 148 (Lynds 1965) is also found in this area, and shows up as a $25^{\prime}$ long filament in [O II], while it appears much shorter in the [O III] emission line image $\left(\sim 18^{\prime}\right)$. Emission in both images is detected further to the 
west of LBN 148 and seems to extend outside our field. We note here that our coverage to the west marginally reaches $\alpha \simeq 19^{\mathrm{h}} 24^{\mathrm{m}}$, while the ROSAT data show that emission extends up to $\alpha \simeq 19^{\mathrm{h}} 23^{\mathrm{m}}$ ( $\mathrm{Lu} \&$ Aschenbach 2002).

In area $\mathrm{F}$ we find a long filamentary structure both in [O II] and [O III] (Figs. 1 and 2) extending from the west edge of our field up to the central areas of the remnant. There is a wealth of short scale structures to the north of this long filament which are only seen in the low ionization image of [O II]. However, further to the north in the [O III] image faint, filamentary and diffuse structures are discovered, around $\alpha \simeq 19^{\mathrm{h}} 27^{\mathrm{m}}$ and $\delta \simeq 33^{\circ} 00^{\prime}$, which seem to extend outside the north boundary of our field and are projected outside the main body of the soft $\mathrm{X}$-ray emission (Lu \& Aschenbach 2002). Based on the extent of the remnant as seen in the radio and $\mathrm{X}$-ray observations it is likely that this emission is not part of $\mathrm{G}$ 65.3+5.7. Substantial differences between the two mosaics are also seen in area G. Although the nature of the [O III] structure observed is filamentary all along the $\sim 42^{\prime}$ of its length, the $[\mathrm{O} \mathrm{II}]$ emission appears quite faint and completely diffuse. Another area with great differences among the low and medium ionization images is area $H$. The structures are much better defined in [O III] than in [O II]. The [O II] image of the area seems to consist of several small scale filamentary structures and some of them do not have [O III] counterparts. Finally, in area I we find that the [O III] emission is better structured compared to the $[\mathrm{O} \mathrm{II}]$ emission line image which, in addition, looks more clumpy. Note that around $\alpha \simeq 19^{\mathrm{h}} 41^{\mathrm{m}}$ and $\delta \simeq 32^{\circ} 20^{\prime}$ faint $[\mathrm{O}$ III $]$ emission is detected in an arc-like shape. Its typical brightness is $\sim 1 / 3$ of the bulk of the emission seen a few arcminutes to the south.

\section{Area $C$ in detail}

Long observing runs would be required in order to map this remnant in the major optical emission lines. The only area observed in more detail is area $\mathrm{C}$ (Fig. 3) which was observed through the lines of $\mathrm{H} \alpha+[\mathrm{N} \mathrm{II}],[\mathrm{S}$ II], [O II] and [O III]. These observations are flux calibrated and show that the emission, in all filters, is strong in absolute terms. However, there are striking differences between the low ionization lines and [O III] also in this area. The major difference being the double filament seen in the center of this field (Fig. 3). It is best defined in the medium ionization line of [O III], while diffuse emission is present in the [O II] image but is hardly detected in the images of $\mathrm{H} \alpha+[\mathrm{NII}]$ and $[\mathrm{S} \mathrm{II}]$. The two filaments are separated by $\sim 1^{\prime}$, while their typical projected thickness is less than $\sim 35^{\prime \prime}$. This angular thickness corresponds to $0.17 \mathrm{pc}$, at a distance of $1 \mathrm{kpc}$, which is much smaller than the total length of this structure of $\sim 19 \mathrm{pc}$. We note here that only a small part $\left(\sim 16^{\prime}\right)$ of this filamentary structure is present in the low ionization images strongly suggesting the absence of complete recombination zones. This seems to be a common characteristic of the various areas of $\mathrm{G} \mathrm{65.3+5.7}$. In order to explore the properties of the double filament, we formed the ratio of the [O II] to the [O III] image. This new image could help to discriminate between projection effects and actual changes in the shock parameters. We find that the measured values of the ratio along each filament change by $\sim 20 \%$, while the average values of the ratios at the two filaments agree to within the $1.5 \sigma$ range. The relatively small difference in the ratio between the two filaments might point to simple projection effects but images of higher spatial resolution would be required to check this issue in more detail.

Finally, we also formed the ratio of the [O III] emission line image to the $\mathrm{H} \alpha+[\mathrm{N} \mathrm{II}]$ image which would allow to establish areas with complete and incomplete recombinations zones (Raymond et al. 1988). Indeed, this image clearly demonstrates that the double filament as well as the strong emission seen in the south-west are characterized by incomplete structures, while the emission from the south-east regions originates from complete shock structures.

\section{The long-slit spectra from G $65.3+5.7$}

We have obtained long-slit spectra at eight different locations of $\mathrm{G} 65.3+5.7$. The absolute $\mathrm{H} \alpha$ flux covers a wide range of values from 0.5 to $22 \times$ $10^{-17} \mathrm{erg} \mathrm{s}^{-1} \mathrm{~cm}^{-2} \operatorname{arcsec}^{-2}$ (Table 2). All spectra suggest that the emission detected during these spectroscopic observations originates from shock heated gas ([S II $] / \mathrm{H} \alpha$ $\sim 0.7-1.8$; Table 2). This conclusion also applies to the single spectrum taken from LBN 148 showing that this object is related to the remnant and is not an HiI region. The ratio of the sulfur lines emitted at $6716 \AA$ and $6731 \AA$ allow us to estimate the electron density (e.g. Osterbrock 1989) at the position observed. The electron densities were calculated with the nebular package within the IRAF software (Shaw \& Dufour 1995). All ratios tend to the high end of the allowable range of values suggesting electron densities below $\sim 150 \mathrm{~cm}^{-3}$ and even below $\sim 30 \mathrm{~cm}^{-3}$. Consequently, we cannot directly measure the preshock cloud density but can only place upper limits. The $\mathrm{H} \alpha / \mathrm{H} \beta$ ratio is used to estimate the logarithmic extinction c towards a source of line emission assuming an intrinsic ratio of 3 . A signal to noise weighted average of the logarithmic extinction $c$ is 4.3 for the different locations around $\mathrm{G} 65.3+5.7$. Higher values are found among the spectra but are also accompanied by larger errors. Fesen et al. (1985) obtained spectra at two locations and found $\mathrm{H} \alpha / \mathrm{H} \beta$ ratios around $3.3( \pm 35 \%)$ in agreement with our measurements within the $1 \sigma$ range.

The most interesting result involves the very large $[\mathrm{O}$ III $] / \mathrm{H} \beta$ ratios observed in most of the spectra. Models of complete shock structures predict values below $\sim 6$ for this ratio (Cox \& Raymond 1985; Hartigan et al. 1987). This limit is easily exceeded in case of shocks with incomplete recombination zones (Raymond et al. 1988). Our measured values for the $[\mathrm{O} \mathrm{III}] / \mathrm{H} \beta$ ratio range from 6 to 110 (Table 2) indicating that shocks with complete and incomplete recombination zones are present. 
Table 2. Relative line fluxes.

\begin{tabular}{lllllllll}
\hline \hline & area 1a & area $1 \mathrm{~b}$ & area $1 \mathrm{c}$ & area 2 & area 3a & area 3b & area 4 & area 5 \\
\hline Line $(\AA)$ & & & & & & & & \\
$4861 \mathrm{H} \beta$ & $22(8)$ & $20(14)$ & $13(5)$ & $20(6)$ & $27(23)$ & $25(26)$ & $22(11)$ & $28(32)$ \\
$4959[\mathrm{O} \mathrm{III}]$ & $240(95)$ & $417(104)$ & $360(103)$ & $128(38)$ & $84(93)$ & $38(33)$ & $52(25)$ & $50(74)$ \\
$5007[\mathrm{O} \mathrm{III}]$ & $757(197)$ & $1273(272)$ & $1074(153)$ & $378(114)$ & $263(166)$ & $123(87)$ & $170(70)$ & $158(173)$ \\
$6300[\mathrm{O} \mathrm{I}]$ & - & - & - & - & $6(18)$ & $16(35)$ & $12(11)$ & $8(11)$ \\
$6364[\mathrm{O} \mathrm{I}]$ & - & - & - & - & - & $6(8)$ & $4(4)$ & $2(3)$ \\
$6548[\mathrm{~N} \mathrm{II}]$ & $30(15)$ & $35(27)$ & $37(15)$ & $40(24)$ & $26(54)$ & $25(44)$ & $37(32)$ & $30(66)$ \\
$6563 \mathrm{H} \alpha$ & $100(39)$ & $100(64)$ & $100(45)$ & $100(60)$ & $100(110)$ & $100(112)$ & $100(77)$ & $100(156)$ \\
$6584[\mathrm{~N} \mathrm{II}]$ & $111(54)$ & $116(71)$ & $130(47)$ & $125(75)$ & $87(109)$ & $82(102)$ & $114(78)$ & $93(152)$ \\
$6716[\mathrm{~S} \mathrm{II}]_{1}$ & $74(32)$ & $87(56)$ & $103(34)$ & $75(50)$ & $74(93)$ & $56(121)$ & $96(74)$ & $61(121)$ \\
$6731[\mathrm{~S} \mathrm{II}]_{2}$ & $55(24)$ & $66(42)$ & $76(25)$ & $56(37)$ & $52(66)$ & $41(88)$ & $67(52)$ & $43(83)$ \\
\hline $\mathrm{Absolute} \mathrm{H \alpha}$ & 6.7 & 9.3 & 10.7 & 20.6 & 16.3 & 22.1 & 10.4 & 17.3 \\
\hline $\mathrm{H} \alpha / \mathrm{H} \beta$ & $4.5(8)$ & $5.0(14)$ & $7.7(5)$ & $5.0(6)$ & $3.7(23)$ & $4.0(26)$ & $4.5(11)$ & $3.6(32)$ \\
$c$ & $0.5(4)$ & $0.7(7)$ & $1.2(5)$ & $0.7(3)$ & $0.28(5)$ & $0.38(7)$ & $0.55(6)$ & $0.24(6)$ \\
{$[\mathrm{O} \mathrm{III}] / \mathrm{H} \beta$} & $45(8)$ & $85(14)$ & $110(5)$ & $25(6)$ & $13(23)$ & $6.4(25)$ & $10(11)$ & $7.4(32)$ \\
{$[\mathrm{S} \mathrm{II}] / \mathrm{H} \alpha$} & $1.29(28)$ & $1.53(47)$ & $1.79(31)$ & $1.31(43)$ & $1.26(90)$ & $0.97(95)$ & $1.63(58)$ & $1.04(106)$ \\
{$[\mathrm{S} \mathrm{II}]_{1} /[\mathrm{S} \mathrm{II}]_{2}$} & $1.34(19)$ & $1.32(34)$ & $1.35(20)$ & $1.34(30)$ & $1.42(54)$ & $1.37(71)$ & $1.43(43)$ & $1.42(68)$ \\
$n_{\mathrm{e}}\left(\mathrm{cm}{ }^{-3}\right)$ & $<150$ & $<140$ & $<140$ & $<130$ & $<45$ & $<70$ & $<35$ & $<30$ \\
\hline
\end{tabular}

\begin{tabular}{lllll}
\hline \hline & area 6 & area 7 & area $8 \mathrm{a}$ & area 8b \\
\hline Line $(\AA)$ & & & & \\
$4861 \mathrm{H} \beta$ & - & $24(18)$ & - & - \\
$4959[\mathrm{O} \mathrm{III}]$ & $135(86)$ & $104(69)$ & $378(31)$ & $690(48)$ \\
$5007[\mathrm{O} \mathrm{III}]$ & $415(239)$ & $326(150)$ & $1265(91)$ & $2040(141)$ \\
$6300[\mathrm{O} \mathrm{I}]$ & $11(13)$ & $7(3)$ & - & - \\
$6364[\mathrm{O} \mathrm{I}]$ & $4(5)$ & - & - & - \\
$6548[\mathrm{~N} \mathrm{II}]$ & $33(39)$ & $27(16)$ & - & - \\
$6563 \mathrm{H} \alpha$ & $100(115)$ & $100(61)$ & $100(9)$ & $100(10)$ \\
$6584[\mathrm{~N} \mathrm{II}]$ & $112(128)$ & $91(56)$ & $135(13)$ & $131(14)$ \\
$6716[\mathrm{~S} \mathrm{II}]_{1}$ & $95(115)$ & $38(23)$ & $93(9)$ & $84(8)$ \\
$6731[\mathrm{~S} \mathrm{II}]_{2}$ & $66(81)$ & $30(19)$ & $89(8)$ & $61(5)$ \\
\hline $\mathrm{Absolute} \mathrm{H} \alpha$ & 7.7 & 9.0 & 0.6 & 0.5 \\
\hline $\mathrm{H} \alpha / \mathrm{H} \beta$ & - & $4.2(17)$ & - & - \\
$c$ & - & $0.44(5)$ & - & - \\
{$[\mathrm{O} \mathrm{III}] / \mathrm{H} \beta$} & - & $18(18)$ & - & - \\
{$[\mathrm{S} \mathrm{II}] / \mathrm{H} \alpha$} & $1.61(88)$ & $0.68(27)$ & $1.82(7)$ & $1.45(7)$ \\
{$[\mathrm{S} \mathrm{II}]_{1} /[\mathrm{S} \mathrm{II}]_{2}$} & $1.44(66)$ & $1.27(15)$ & $1.04(6)$ & $1.38(4)$ \\
$n_{\mathrm{e}}\left(\mathrm{cm}{ }^{-3}\right)$ & $<30$ & $<170$ & & \\
\hline & & & & \\
\hline
\end{tabular}

The numbers next to the areas number indicate different apertures extracted along the slit. Listed fluxes are a signal to noise weighted average of the individual fluxes and are not corrected for interstellar extinction. Numbers in parentheses represent the signal to noise ratio of the quoted fluxes. Absolute $\mathrm{H} \alpha$ flux in units of $10^{-17} \mathrm{erg} \mathrm{s}^{-1} \mathrm{~cm}^{-2} \operatorname{arcsec}^{-2}$. All relative fluxes normalized to $F(\mathrm{H} \alpha)=100$.

Fesen et al. (1985) measured this ratio to be $\sim 2$ (their position 1 ) and $\sim 40$ (their position 2 ). Our area 1 lies close to their position $2\left(\sim 90^{\prime \prime}\right.$ apart $)$ and there the measured ratios of $[\mathrm{O} \mathrm{III}] / \mathrm{H} \beta$ are 46 and 86 along the slit (Table 2). These spectra were taken at different positions and thus, cannot be directly compared but they do point to the presence of incomplete recombination zones in this part of the remnant. We note here that the signal to noise ratios quoted in Table 2 do not include calibration errors which are less than $\sim 10 \%$.

As is evident from Table 2 only a small number of the extracted spectra points to shocks with complete 
recombination zones. The spectra from positions $1,2,3 \mathrm{a}$, $4,6,7$, and 8 strongly support the presence of incomplete recombination zones $([\mathrm{O} \mathrm{III}] / \mathrm{H} \beta \sim 10-100)$. Examination of the theoretical estimates of the $[\mathrm{O}$ III $] / \mathrm{H} \beta$ and $[\mathrm{O} \mathrm{I}] / \mathrm{H} \beta$ ratios as a function of column density (Raymond et al. 1988) shows that shock speeds higher than $\sim 100 \mathrm{~km} \mathrm{~s}^{-1}$, most likely around $120 \mathrm{~km} \mathrm{~s}^{-1}$, and column densities in the range of $10^{17}-10^{18.5} \mathrm{~cm}^{-2}$ are required to match the observations. Especially, at position 1 a shock velocity of $120 \mathrm{~km} \mathrm{~s}^{-1}$ and a column density of $\sim 10^{17.5} \mathrm{~cm}^{-2}$ is estimated, which is well below that of $\sim 10^{18.7} \mathrm{~cm}^{-2}$ required for a complete recombination zone.

\section{Discussion}

The extended remnant G $65.3+5.7$ was observed for the first time with a highly efficient CCD camera in the low and medium ionization lines of [O II] $3727 \AA$ and [O III $] 5007 \AA$, respectively. The [O II] interference filter being in the far blue part of the optical wavelength band provides sharp images since the emission from field stars is significantly reduced, while any morphological differences between the two images can not be attributed to abundance variations. In addition, we performed the first flux calibrated imaging observations of a specific area in the south of G $65.3+5.7$ (area C, Sect. 4) in major optical emission lines. These observations as well as the raster observations reveal in sufficient detail what is already known for parts of the remnant, i.e. strong differences between the lower and higher ionization lines (Rosado 1981; Fesen et al. 1983; Sitnik et al. 1983).

The object under study is an excellent example of the class of remnants displaying prominent [O III] emission and is this line that provides the sharpest view of the system (e.g. Fesen et al. 1997; Mavromatakis et al. 2002). In the following we will assume a distance of $1 \mathrm{kpc}$ to the remnant and an angular radius of 1.8 implied by the $\mathrm{X}$-ray ( $\mathrm{Lu} \&$ Aschenbach 2002) and our optical data. The thinnest filamentary structures seen in the [O II] images are characterized by typical projected widths, actually the full width at half maximum (fwhm), of $\sim 20^{\prime \prime}$ or $0.1 \mathrm{pc}$, while the shortest filaments are $\sim 0.5 \mathrm{pc}$ long. The longest ones are of the order of several pc. We also find gaps in certain filaments which otherwise look continuous. The shortest gaps are typically around $0.2 \mathrm{pc}$ suggesting this length as the typical length of small scale inhomogeneities in this cloudy environment.

A shock velocity of $\sim 120 \mathrm{~km} \mathrm{~s}^{-1}$ and a column density of $\sim 10^{17.5}-10^{18} \mathrm{~cm}^{-2}$ are estimated for area 1 from the optical spectra and the modeling results of Raymond et al. (1988). The measured fwhm of $0.14 \mathrm{pc}$ of the filament in this area and the estimated column density allow us to estimate an average filament density of $\sim 0.8-2.5 \mathrm{~cm}^{-3}$. The actual preshock cloud density must be lower than this by, at least, a factor of 4. An IUE spectrum taken a few arcseconds off our position shows emission from C III], N III], O III], but weak C IV and maybe Si IV, and O IV (Raymond J. 2002, private communication). Thus, it is not possible to fully explore the physical conditions in this area as Raymond et al. (1988) have done for a bright filament in the Cygnus Loop. Using the same approach to other areas of the remnant we find average densities in the range of $\sim 0.3-4 \mathrm{~cm}^{-3}$. Specifically, for area 5 a shock velocity of $90 \mathrm{~km} \mathrm{~s}^{-1}$ and column densities in the range of $10^{18}-10^{18.5} \mathrm{~cm}^{-2}$ are estimated. Extraction of the intensity profiles from the [O II] and [O III] images along the declination axis shows that the positions of maximum intensity differ by $\sim 0.07 \mathrm{pc}$ with the $[\mathrm{O}$ III] profile leading, while the fwhm of the fitted Gaussian profiles are 0.13 and $0.19 \mathrm{pc}$, respectively. This behaviour of the lower and higher ionization lines is also verified by the long slit spectra which are characterized by a spatial scale of $1 / 350 \mathrm{pc}$ compared to that of $1 / 40 \mathrm{pc}$ of the wide field images. Assuming a typical length of $0.19 \mathrm{pc}$ and a column density of $\sim 10^{18.3} \mathrm{~cm}^{-2}$, we estimate the average density behind the radiative shock as $\sim 3.5 \mathrm{~cm}^{-3}$. These simplified calculations show that in several areas of the remnant the particle density in the recombination region is of the order of a few atoms per $\mathrm{cm}^{3}$.

In areas where complete recombinations zones are suggested by the observational data we can utilize the relations

$n_{[\mathrm{S} \mathrm{II}]} \simeq 45 n_{\mathrm{c}} V_{\mathrm{s}}^{2}$

and

$E_{51}=2 \times 10^{-5} \beta^{-1} n_{\mathrm{c}} V_{\mathrm{s}}^{2} r_{\mathrm{s}}^{3}$,

given by Fesen \& Kirshner (1980) and McKee \& Cowie (1975), respectively, to limit the preshock cloud density and explosion energy. The factor $\beta$ is of the order of $1-2$, $n_{[\mathrm{S} \mathrm{II}]}$ is the electron density derived from the sulfur line ratio, $n_{\mathrm{c}}$ is the preshock cloud density, $E_{51}$ is the explosion energy in units of $10^{51} \mathrm{erg}, V_{\mathrm{s}}$ is the shock velocity into the clouds in units of $100 \mathrm{~km} \mathrm{~s}^{-1}$, and $r_{\mathrm{s}}$ is the radius of the remnant in pc. Assuming an upper limit of $\sim 70 \mathrm{~cm}^{-3}$ on the electron density (Table 2 ; areas $3 \mathrm{~b}, 4,5$ ) we find that $n_{\mathrm{c}} V_{\mathrm{s}}^{2}<1.6$ and consequently, $E_{51}<1$ for $r_{\mathrm{s}}$ $\simeq 32$ and $\beta \simeq 1$. Although this limit is rather broad, it is consistent with the explosion energy of 0.2 estimated by Lu \& Aschenbach (2002) from the Sedov-Taylor solution of the X-ray data. However, in order to derive Eqs. (1) and (2) it is assumed that the magnetic field component perpendicular to the shock velocity vector is negligible (see also Bocchino et al. 2000). If this is not the case then the preshock cloud density will be expected to be higher than what Eq. (1) implies. Comparison of the shock velocities estimated from the optical data with the shock velocity of the primary blast wave suggests density contrasts $\sim 10$ 20 between the interstellar medium and the interstellar clouds. The preshock medium density of $0.02 \mathrm{~cm}^{-3}$ given by Lu \& Aschenbach (2002) implies typical preshock cloud densities $\sim 0.2-0.4 \mathrm{~cm}^{-3}$, which are consistent with the upper limits obtained earlier.

The observed $(\mathrm{H} \alpha / \mathrm{H} \beta)_{\text {obs }}$ ratio can be used to estimate the logarithmic extinction $c$ towards a source of 


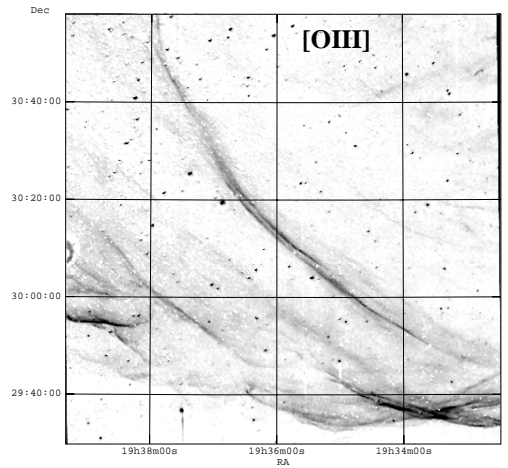

(a)

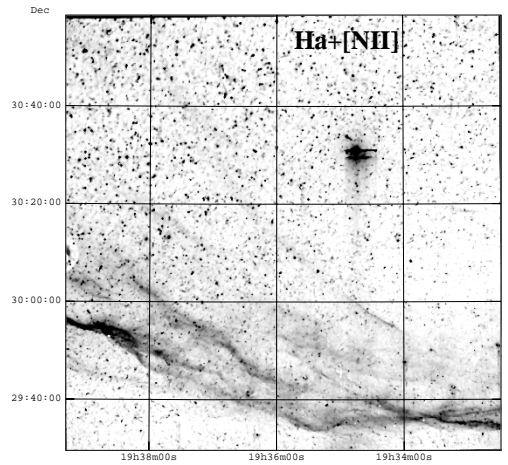

(c)

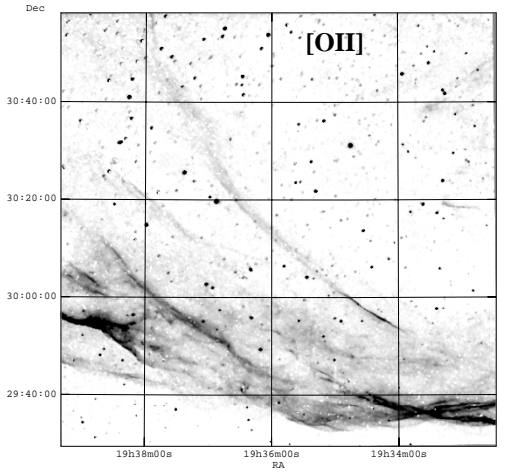

(b)

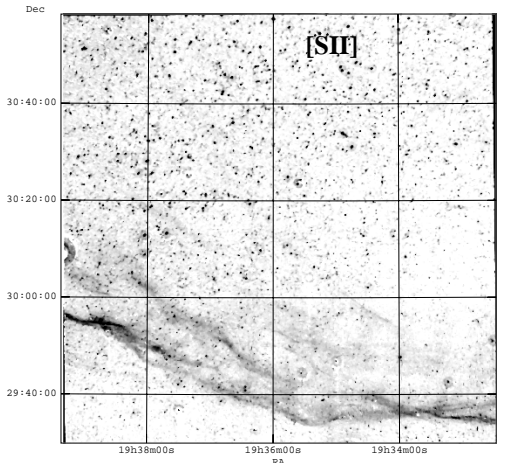

(d)

Fig. 3. A 1.5 wide area in the south of G $65.3+5.7$ imaged with the $[\mathrm{O}$ III $] 5007 \AA$ a), [O II $] 3727 \AA$ b), H $\alpha+[\mathrm{N}$ II $]$ c) and [S II] d) filters. The images have been smoothed to suppress the residuals from the imperfect continuum subtraction. The shadings run linearly from 0 to 75 in Fig. 3a, 0 to 40 in Fig. 3b, 0 to 80 in Fig. 3c and 0 to 30 in Fig. 3d in units of $10^{-17} \mathrm{erg} \mathrm{s}^{-1} \mathrm{~cm}^{-2}$ arcsec ${ }^{-2}$.

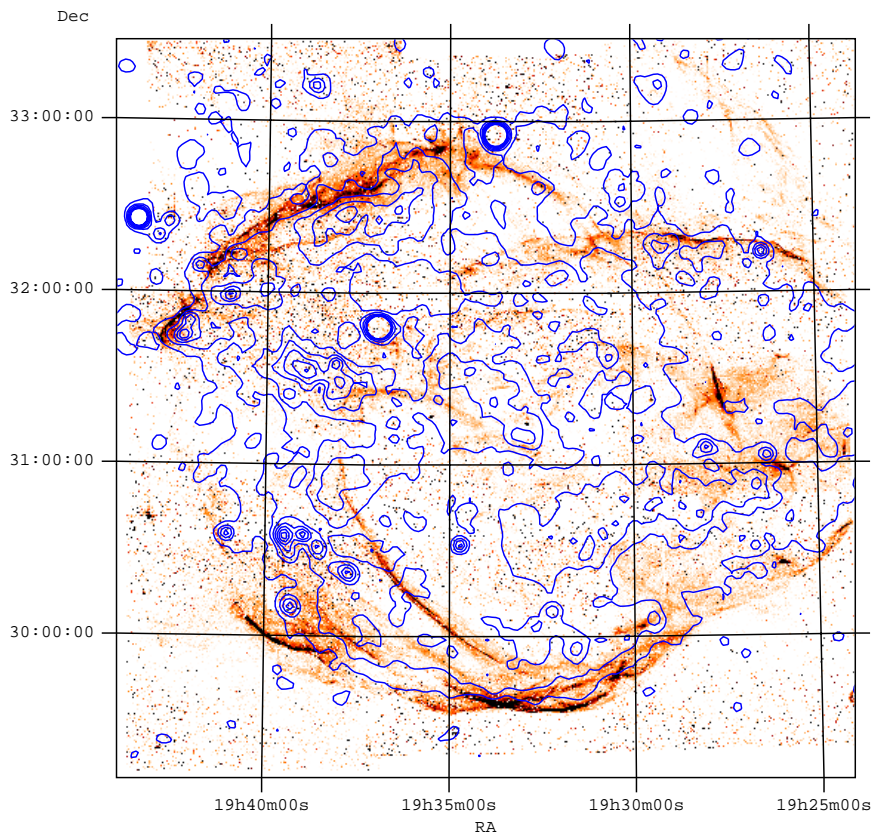

Fig. 4. The $[\mathrm{O}$ III $]$ emission of $\mathrm{G} 65.3+5.7$ shown together with the soft X-ray emission detected from ROSAT (Lu $\&$ Aschenbach 2002). The $\mathrm{X}$-ray emission is shown in contours starting from $2.5 \times 10^{-4} \mathrm{cts} \mathrm{s}^{-1}$ pixel $^{-1}$ in steps of $3.5 \times 10^{-4} \mathrm{cts} \mathrm{s}^{-1}$ pixel $^{-1}$, while each pixel is $0^{\prime} .75 \times 0^{\prime} .75$.

line emission. The relation we use is $c=1 . / 0.331 *$ $\log \left((\mathrm{H} \alpha / \mathrm{H} \beta)_{\mathrm{obs}} / 3\right.$. $)$ and is based on the interstellar extinction curve of Kaler (1976) as implemented in the nebular package (Shaw \& Dufour 1995) within the IRAF software. The minimum extinction of $0.24( \pm 0.04)$ is measured in area 5 , while the maximum, statistically significant measurement of $0.8( \pm 0.2)$ is found in area 1a. The absorption at area 1 and 2 is higher than in other parts of the remnant. This may be related to the presence of excess emission seen in the IRAS $60 \mu \mathrm{m}$ maps (Wheelock et al. 1994) to the east of $19^{\mathrm{h}} 40^{\mathrm{m}}$ provided that the dust emission lies to the foreground of $\mathrm{G} 65.3+5.7$. The observed variations of the logarithmic extinction may indicate the presence of intrinsic absorption although these variations are at the 3-4 $\sigma$ level. Note that variations in the column density, determined from X-ray measurements, are reported by $\mathrm{Lu} \&$ Aschenbach (2002). The probable non-uniform distribution of the column density and the logarithmic extinction would suggest a complex, inhomogeneous environment into which the primary blast wave propagates. In order to estimate the column density implied by the optical spectra we use the relation $N_{\mathrm{H}}=5.4( \pm 0.1) \times 10^{21} E(B-V) \mathrm{cm}^{-2} \mathrm{mag}^{-1}$ (Predehl $\&$ Schmitt 1995). Assuming a logarithmic extinction of $0.36( \pm 0.03)$, the average from areas 3,4 , and 5 , which is equivalent to an $E(B-V)$ of $0.24(E(B-V)=0.664 c$; Kaler 1976; Aller 1984), a column density of $13( \pm 1)$ $\times 10^{20} \mathrm{~cm}^{-2}$ is calculated. This column density exceeds that of $3-5 \times 10^{20} \mathrm{~cm}^{-2}$ measured in the ROSAT pointed data (Lu \& Aschenbach 2002) by factors in the range of 
2.6-4.3. It is not clear at the moment if this implies the presence of excess intrinsic absorption affecting mainly the optical wavelengths or is due to a statistical effect since these factors are just compatible with the scattering around the straight line fit of Predehl \& Schmitt (1995).

The supernova remnant G $65.3+5.7$ was observed in the soft $\mathrm{X}$-ray band by ROSAT through a large number of pointed observations as well as during the AllSky survey (Lu \& Aschenbach 2002). In Fig. 4 we show the [O III] emission together with the contours of the soft $\mathrm{X}$-ray emission detected by ROSAT. The outer [O III] filaments are rather well correlated with the $\mathrm{X}$-ray emission mainly in the south, and north, north-east areas. The southern [O III] filaments probably lie close to the primary shock wave, while $\mathrm{X}$-ray emission is detected ahead of the northern [O III] filaments. The clumpiness of the $\mathrm{X}$-ray emission in the central areas of the remnant does not allow a reliable comparison with the optical emission. The temperature or column density maps do not provide any clear evidence for a correlation or anticorrelation with the optical data. There is also no well defined $\mathrm{X}$-ray emission outside the shell of the remnant around $\alpha \simeq 19^{\mathrm{h}} 28^{\mathrm{m}}, \delta \simeq$ $33^{\circ} 00^{\prime}$ suggesting that the detected optical emission may not be related to it.

\section{Conclusions}

The supernova remnant G $65.3+5.7$ was observed in the lower and medium ionization lines of $\left[\mathrm{O}_{\mathrm{II}}\right] 3727 \AA$ and [O III $] 5007 \AA$. The [O III] emission line mosaic is more filamentary than the $[\mathrm{OII}]$ image. Several new structures are detected, while some of them are found outside the $\mathrm{X}$-ray shell indicating that those may not be related to $\mathrm{G} 65.3+5.7$. The morphology of the $[\mathrm{O} \mathrm{II}]$ and [O III] images suggest the presence of small scale inhomogeneities which are of the order of $\sim 0.2$ pc. Larger scale inhomogeneities are also present. The long slit spectra clearly demonstrate the very strong [O III] emission relative to $\mathrm{H} \beta$ suggesting the presence of incomplete recombination zones. The shock velocities are found in the range of $90-140 \mathrm{~km} \mathrm{~s}^{-1}$, while the column densities behind the radiative shock cover the range of $\sim 10^{17.0}-10^{18.5} \mathrm{~cm}^{-2}$. The extinction variations seen in the optical spectra may indicate the presence of internal absorption.

Acknowledgements. The authors would like to thank J. Raymond and the referee B. Aschenbach for their useful comments and suggestions. Skinakas Observatory is a collaborative project of the University of Crete, the Foundation for Research and Technology-Hellas and the Max-Planck-Institut für Extraterrestrische Physik. This research has made use of data obtained through the High Energy Astrophysics Science
Archive Research Center Online Service, provided by the NASA/Goddard Space Flight Center.

\section{References}

Aller, L. H. 1984, Physics of thermal gaseous nebulae (D. Reidel Publishing Company)

Aschenbach B. 1994, ROSAT observations of supernova remnants, in Proc. of New Horizon of X-ray Astronomy, ed. F. Makino, \& T. Ohashi (Universal Academy Press), 103

Bocchino, F., Maggio, A., Sciortino, S., \& Raymond, J. 2000, A\&A, 359, 316

Cox, D. P., \& Raymond, J. C. 1985, ApJ, 298, 651

Fesen, R. A., \& Kirshner, R. P. 1980, ApJ, 242, 1023

Fesen, R. A., Gull, T. R., \& Ketelsen, D. A. 1983, ApJS, 51, 337

Fesen, R. A., Blair, W. P., \& Kirshner, R. P. 1985, ApJ, 292, 29

Fesen, R. A., Winkler, P. F., Rathore, Y., et al. 1997, AJ, 113, 767

Gorham, P. W., Ray, P. S., Anderson, S. B., Kulkarni, S. R., \& Prince, T. A. 1996, ApJ, 458, 257

Gull, T. R., Kirshner, R. P., \& Parker, R. A. R. 1977, ApJ, 215, L69

Hartigan, P., Raymond, J., \& Hartmann, L. 1987, ApJ, 316, 323

Kaler, J. B. 1976, ApJS, 31, 517

Lasker, B. M., Russel, J. N., \& Jenkner, H. 1999, in the HST Guide Star Catalog, version 1.1-ACT, The Association of Universities for Research in Astronomy, Inc.

Lu, F. J., \& Aschenbach, B. 2002, to be submitted

Lynds, B. T. 1965, ApJS, 12, 163

Mason, K. O., Kahn, S. M., Charles, P. A., Lampton, M. L., \& Blisset, R. 1979, ApJ, 230, L163

Mavromatakis, F., Papamastorakis, J., Paleologou, E. V., \& Ventura, J. 2000, A\&A, 353, 371

Mavromatakis, F., Boumis, P., \& Paleologou, E. V. 2002, A\&A, 383,1011

McKee, C. F., \& Cowie, L. 1975, ApJ, 195, 715

Osterbrock, D. E. 1989, Astrophysics of gaseous nebulae (W. H. Freeman \& Company)

Predehl, P., \& Schmitt, J. H. M. M. 1995, A\&A, 293, 889

Raymond, J. C., Hester, J. J., Cox., D., et al. 1988, ApJ, 324, 869

Reich, W., Kallas, E., \& Steube, R. 1979, A\&A, 78, L13

Rosado, M. 1981, ApJ, 250, 222

Sabbadin, F., \& D'Odorico, S. 1976, A\&A, 49, 119

Seward, F. 1990, ApJS, 73, 781

Shaw, R. A., \& Dufour, R. J. 1995, PASP, 107, 896

Sitnik, T. G., Klementéva, A. Y., \& Toropova, M. S. 1983, Sov. Astr., 27, 292

Snyder, W. A., Davidsen, A. F., Henry, R. C., et al. 1978, ApJ, 222, L13

Wheelock, S. L., Gautier, T. N., Chillemi, J., et al. 1994, IRAS sky survey atlas, Explanatory supplement 